\title{
FRAMEWORK FOR END-TO-END QOS MEASUREMENT OVER DVB-RCS NETWORK
}

\author{
M. Ali, L. Liang, Z. Sun (Member IEEE) and H. Cruickshank (Member IEEE) \\ Centre for Communication Systems Research, University of Surrey, Guildford, Surrey, GU2 7XH, UK \\ \{m.ali, l.liang, z.sun, h.cruickshank@surrey.ac.uk\}
}

Keywords: QoS, DVB-RCS, IPv4, IPv6, Satellite network.

\begin{abstract}
Satellites are popular due to their wide area coverage and for providing connectivity in remote regions of the world. The future development of satellite systems providing services based on the Internet Protocol (IP) needs to be validated on a real satellite network. This paper presents the end-to-end quality of service (QoS) measurements taken at European Space Agency (ESA) testbed over DVB-RCS infrastructure.

The applications chosen for these experiments are file transfer (FTP), web browsing (HTTP), video streaming and P2P filesharing. File transfer, web browsing and P2P file-sharing require reliable transport mechanism as a corrupted bit will hinder the intact data delivery. Therefore, these applications use transmission control protocol (TCP) as the transport protocol. TCP involves a three way handshake, which introduces extra delay during data transfer. Video streaming is a real time application, so, it is time-sensitive and requires lesser reliability compared to the other three applications. Hence, it employs user datagram protocol (UDP) at the transport layer, which do not offer any guarantee of reliable data delivery but is fast. The parameters that have been used to evaluate quality of service (QoS) are packet timestamps, file download time, round trip delay, packet sizes and packet loss rate.

Also similar applications and results will be measured from a satellite emulation testbed, PLATINE. It is based on Linux operating system, in which most of the DVB-S and DVBRCS satellite network functions have been implemented. These functions include network topology configuration, Quality of Service (QoS), Demand Assigned Multiple Access (DAMA), traffic encapsulation using both Asynchronous Transfer mode (ATM) and ULE/MPEG, satellite network entities configuration and support for both IPv4 and IPv6.

The paper concludes with the comparative analysis of the QoS of the applications in both real and emulation environments.
\end{abstract}

\section{Introduction}

Satellite systems are integrating with the global information infrastructure as one of the major technologies providing both television broadcast services and Internet access. These systems are supporting most of the same applications, services, and protocols as the terrestrial networks. Standards for satellite systems are required to optimize for Internet access. Unified satellite standards will finish the dominance of proprietary solutions, allowing penetration in telecommunications commercial market [7]. In urban areas, Internet access is available via terrestrial networks, whereas, infrastructure deprived rural areas rely on wireless connectivity for this facility. It takes a long time to develop terrestrial networks to rural areas. So, satellites can offer Internet access to such remote regions.

Satellites are characterized by their long propagation delay; hence, quality of service (QoS) is an issue to be handled in these networks. In this paper, we are presenting the end-toend QoS measurement plan in DVB-RCS environment. The applications to be tested are file transfer (FTP), web browsing (HTTP), video streaming and P2P file-sharing. These applications will be tested on both, IPv4 and IPv6 network protocols for comparative analysis. The different QoS parameters considered are packet timestamps, file download time, round trip delay, packet sizes and packet loss rate.

This paper is divided into the following sections: Section 2 is an overview of DVB-RCS standard; Section 3 is a description of IPv6 and its deployment issues in satellites; Section 4 describes the emulated satellite network testbed at CCSR; Section 5 describes the real ESA testbed used to perform the experiments; the different applications considered and the experimental plan have been briefly reviewed in Section 6; and finally, Section 7 concludes the paper.

\section{DVB-RCS Standard}

The DVB Project (digital video broadcasting, DVB) started the development of a system for digital television broadcasting via satellite (DVB-S) [11] in 1992 and finalized the specification in 1993 [8]. Over the last years, the DVB-S has become the preferred format for satellite broadcasting worldwide. Initially, to support Internet services for DVB-S, the return channel used terrestrial networks. The emergence of digital video broadcast-return channel via satellite (DVBRCS) [12] transformed the one-way DVB-S system into an interactive system and eliminated cables in the return path for Internet.

The DVB-RCS standard was developed in 1997 and finally accepted as a European Telecommunications Standards Institute (ETSI) specification in March 2000. In September 2001, the guideline document was published. Early trial 
products were tested in 2000-2002, and the systems started getting stable, mature and widely acceptable during 2002. Satellite standardization organizations, operators, and manufacturers are collaborating and striving over the last years to achieve a viable solution for interactive services via satellite [7].

In the forward link, DVB-RCS is based on the DVB-S standard. It employs time-division multiplexing at a data rate up to tens of megabits per second. In order to provide Internet access and interactivity, it uses the existing moving pictures expert group-2 (MPEG-2) transport stream to encapsulate IP packets. This encapsulation is performed according to the DVB specification.

The return link in DVB-RCS employs a multiple-frequency time-division multiple-access (MF-TDMA) scheme. It allows a group of terminals to communicate using a set of carrier frequencies, each of which is divided into time slots. The allocation mechanism is based on the statistical multiplexing principle, to utilize the satellite bandwidth optimally. This allocation is managed from the network control centre (NCC) at the gateway. The NCC is responsible for synchronization of the system, via the network clock reference (NCR). It transmits a number of specific system tables in order to update the terminals about issues related to quality of service (QoS), service level agreement (SLA), bandwidth allocation, etc.

\section{IPv6 and Satellites}

The next generation network protocol, IPv6, is emerging to overcome the shortcomings of IPv4, the most important being the shortage of address space [9]. Several Internet Service Providers (ISPs) have offered IPv6 services in North America, Europe and Japan. Because of its huge address space, it will be adapted by mobile and vehicular networks. Although, IPv6 is proliferating and integrating in the existing networks but it has to coexist with IPv4 using dual-stack, protocol translation and tunnelling approaches for a long transitional period [9].

Trial deployments have demonstrated that IPv6 can be used over satellites [2]. Current Digital Video Broadcast (DVB) standards do not document much about satellite for IPv6. The default Multi-Protocol Encapsulation (MPE) cannot deploy IPv6 due to lack of source MAC address, and a payload Type fields. In absence of IPv6 support, tunnel mode (e.g. IPv6 over IPv4), or a link layer encapsulation (LLC), can be used. These schemes add extra processing time. IPv6-friendly encapsulation schemes, like, Unidirectional Lightweight Encapsulation (ULE) encapsulates the packets in the MPEG2 Transport Stream. The introduction of Generic Streams by DVB-S2 makes necessary a new encapsulation, the Generic Stream Encapsulation (GSE) that relies in some fundamental design choices of ULE. The next generation satellite system has to be designed to be as far as compatible with GSE/ULE. However, as vast majority of existing satellite systems use MPE, the transition may take a long time.

The commercial two-way DVB-RCS terminals do not fully support IPv6. IPv6 over DVB-RCS has been tested in some projects (e.g., the EC SATSIX Project [13]), highlighting the hindrances. An upgrade to IPv6 needs some amendments in the standards, especially to ensure that control and management planes are IPv6-enabled.

\section{Satellite Network Testbed}

The satellite network testbed at Centre for Communication Systems Research (CCSR) in University of Surrey consists of one switch and six Linux servers. The servers are named grumpy, sneezy, sleepy, happy, bashful and dopey. Sneezy, sleepy and happy emulate a satellite gateway and two satellite terminals respectively as shown in Fig. 1. A laptop with satellite emulator software, Platine [1], is connected to the switch. Now, this laptop, gateway and the two satellite terminals emulate a satellite network as depicted in Fig. 1. Grumpy, bashful and dopey are three clients where users can install their software for testing the network. After starting the emulator the clients are at a one-way delay of $250-270 \mathrm{~ms}$ from each other.

Platine [1] is a satellite emulator, used in the IST SATSIX project [13] to test and demonstrate various features of satellite networking. Many real satellite networks do not allow performing experiments on their infrastructure, so emulators can be used as alternatives for those real networks. Platine is designed and developed to replicate a real DVBRCS (Digital Video Broadcasting - Return Channel via Satellite) / DVB-S2 (Digital Video broadcasting - Second). It can be configured as a satellite hub or a regenerative payload with onboard processing.

Sneezy works as a gateway or network control centre (NCC). The gateway broadcasts a signalling frame at regular intervals, the terminal burst time plan (TBTP), which updates the timeslot allocation within a superframe between every competing satellite terminal (ST). This allocation is managed by the protocol, Demand Assigned Multiple Access (DAMA). The capacity types that can be allocated are continuous rate assignment (CRA), rate-based dynamic capacity (RBDC), volume-based dynamic capacity (VBDC) and free capacity assignment (FCA). CRA is the basic capacity corresponding to a guaranteed rate which is allocated to the terminal whether the terminal has traffic to send or not. RBDC and VBDC are on demand, and FCA is the plus, lest of available unused

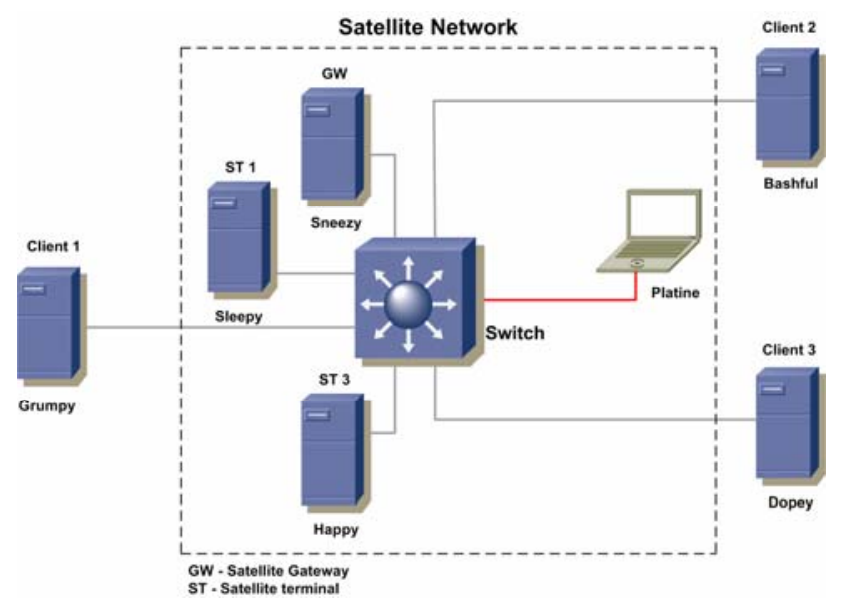

Fig. 1. Satellite network testbed 


\begin{tabular}{|l|l|}
\hline Parameter & Value \\
\hline Uplink encapsulation scheme & ATM_AAL5 \\
\hline Downlink encapsulation scheme & MPEG_ATM_AAL5 \\
\hline Satellite type & Regenerative \\
\hline Delay & $250 \mathrm{~ms}$ \\
\hline Bit error rate (BER) & 0 \\
\hline DVB Scenario & Individual \\
\hline Frame duration & $50 \mathrm{~ms}$ \\
\hline Second duration & $1000 \mathrm{~ms}$ \\
\hline No. of frames per superframe & 2 \\
\hline Carrier transmission rate & $1024 \mathrm{kbps}$ \\
\hline Number of carriers & 2 \\
\hline
\end{tabular}

Table 1: Satellite emulator configuration.

capacity. Any satellite terminal can be allocated one or blend of the four capacities.

Diffserv [3] is the model of QoS in most satellite systems; hence, it is adopted by Platine. This standard defines five perhop behaviour (PHB) classes: expedited forwarding (EF) and four assured forwarding (AF) classes, along with the best effort (BE) service of the Internet. In satellites, typically, higher priority classes of service (e.g., EF and AF) are linked with assured capacity (CRA, RBDC), while lower priority classes (e.g., BE) are primarily specified best effort capacity (VBDC, FCA).

Sleepy and happy are the satellite terminals (ST), which receive the TBTP from sneezy (NCC). The allocations from the NCC are stored by them and used in the next bandwidth allocation requests.

The different parameters configured in the satellite emulator are tabulated in Table 1.

\section{ESA Testbed}

The European Space Agency (ESA) testbed is shown in Fig. 2. It consists of a gateway and two satellite terminals. HellasSat is the main satellite to offer connectivity among them. Different applications and servers can be installed on the local area networks (LAN) connected to the gateway and the terminals.

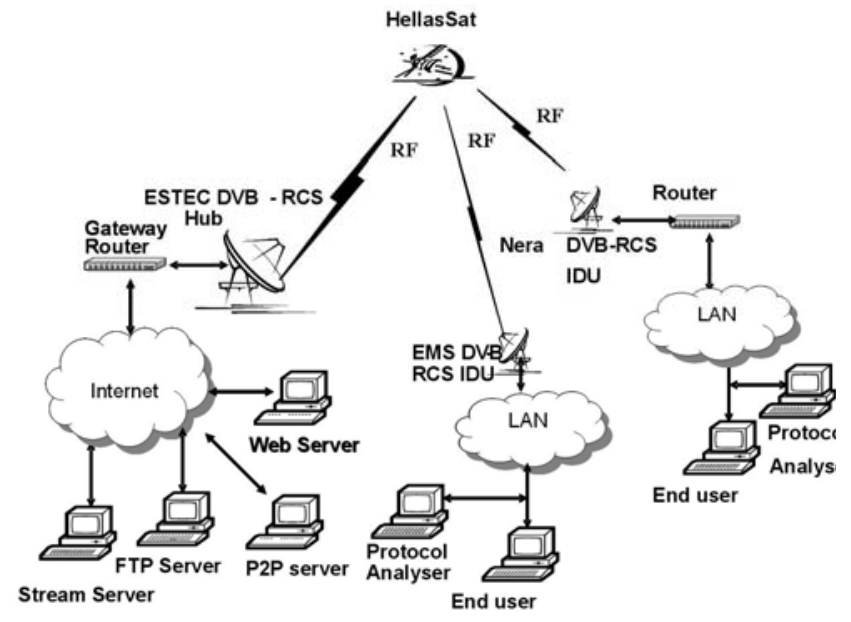

\section{Experimental Plan}

Different applications that will be tested on the emulated and real testbed are described in the following subsections. The softwares to be installed on the clients and servers are also described.

\subsection{File transfer}

File Transfer Protocol (FTP) is a method of downloading files from and uploading files to another system using TCP over a network. It operates in a client/server paradigm. FTP uses two separate connections to send files. One TCP connection is used to send control messages, such as user identification, password, etc., and the other is used to send data. As FTP sends the control messages separately, so it is referred as an out-of-band protocol.

A client utility (ftp) invokes the file transfer. In addition to the original ftp utility, there are many textual and graphical FTP client programs, including most browsers, which run under different operating systems. An FTP utility that supports both IPv4 and IPv6 is lftp [14]. We will use this client program for the end-to-end QoS measurements. There are also many FTP server programs. One of the available servers included in Linux is Very Secure FTP daemon (vsftpd) package. It can be configured for both IPv4 and IPv6.

\subsection{Web browsing}

After telephony, World Wide Web (WWW) is the application that revolutionized the human interaction. It is based on the HyperText Transfer Protocol (HTTP). It also works in a client/server model. The client and server programs exchange HTTP messages to transfer web content. The client program is the browser that opens the Web pages and offers other features like playing audio and video, uploading and downloading files, filling forms, etc. Popular Web browsers are Firefox, Netscape, lynx and Internet Explorer. On the server side, the Web server, houses the web content. HTTP uses TCP as the transport protocol. One of the worldwide deployed, Linux based Web server is Apache. In its latest versions, if support for IPv6 is available in the operating system, its IPv6 listening sockets open by default [4].

\subsection{Video streaming}

In this class of applications, clients request on-demand compressed video files that are stored on servers. A client typically begins playout of the video a few seconds after it begins receiving the file from the server. The client will be playing out video from one location in the file while it is receiving later parts of the file from the server. Both TCP and UDP are used in video streaming.

One of the amazing media players is VLC [16]. It is a lightweight media player that can play numerous video formats. It is supported by Windows, Linux and PocketPC/WinCE handhelds. It can be used as a streaming server supporting both IPv4 and IPv6.

Fig. 2. ESA testbed 


\subsection{P2P file-sharing}

P2P file sharing accounts for more traffic than any other application. It is evolving content distribution architecture because ordinary peers are exchanging the content, bypassing the third-party servers. P2P file-sharing is highly scalable because it relies on the resources of distributed peers around the globe. It is not dependent on proprietary hosted content. It also works on client/server model but each peer plays the role of both, the client and server, at the same time. It uses TCP to ensure reliable transfer of web content. There are many P2P file-sharing applications like BitTorrent, Kazaa, eMule, etc. BitTornado [10] is an extension of BitTorrent, with advanced feature of IPv6 support.

\subsection{Performance metrics}

The performance of different applications described, will be measured for IPv4 and IPv6, using the satellite network testbed at CCSR and ESA testbed. Wireshark [17], formerly known as ethereal, a packet analyzer is used to capture packets at the client and server to measure and calculate different performance metrics. The end-to-end QoS parameters to be measured will be packet timestamps, file download time, round trip delay, packet sizes and packet loss rate.

Packet timestamps are used to measure jitter. Jitter is the variation in delay of consecutive packets. It is due to the variable time elapsed in the queues of the routers in the path. The delay experienced in router queue is dependent on the scheduling policy of the router and the congestion in the network.

Delay is the transit time from the client to the server observed by the packets. In satellites, this time is dominated by the propagation delay. In addition to this, other factors adding to the delay are router queues and number of hops in the path. A simple way to calculate the delay is to synchronize the client and the server and calculate the time difference.

Packet size affects different applications. Larger packets experience more packet loss and delay as compared to smaller packets. Smaller packets are vulnerable to higher jitter in contrast with larger ones.

Packet loss occurs due to network congestion and buffer overflow at routers. It can be reduced by retransmission or error recovery mechanisms. Some applications cannot tolerate packet loss beyond a certain threshold.

Network Time Protocol (NTP) [5] synchronizes computer clocks and promises a precision of 1 microsecond. Most of the measurements in our experiment are related to time, so both the client and server should be synchronized. To meet this requirement, we will install NTP server and synchronize the systems with its clock.

\section{Summary}

DVB-RCS technologies has been studied for years in the satellite community and many research efforts have been put to establish such a system with optimized radio resource management and efficient interworking with other IP networks. This paper presented a test plan sponsored by the
SATNEX project to measure the performance of such a DVBRCS system. The measurement test will be launched on both a DVB-RCS emulator testbed located in the University of Surrey in UK and a real DVB-RCS satellite network hosted by ESA so the results can be verified and calibrated between two measurements. Four widely used Internet applications will be used in the measurement to make the results general for daily network usages. The measurement is going to be carried out in a very short future.

\section{Acknowledgements}

The authors acknowledge the support by the EU FP6 SATNEX project.

\section{References}

[1] C. Baudoin, et. al., "PLATINE: DVB-S2/RCS enhanced testbed for next generation satellite networks" IP Networking over NextGeneration Satellite Systems, International Workshop, Budapest, (2007).

[2] Dr. G. Fairhurst, "IPv6 - The Network Protocol of the Future," IEEE Advanced Satellite Mobile Systems, (2008).

[3] D. Grossman, "New Terminology and Clarifications for Diffserv", RFC 3260, (2002).

[4] I. Hadid and D. Gordon, "Apache Talking IPv6", Linux Journal, (2003).

[5] David L. Mills, “NTP: Network Time Protocol (NTP) Version 3 Specification, Implementation and Analysis,” RFC 1305, Mar. (1992).

[6] H. Schulzrinne, S. Casner, R. Frederick, and V. Jacobson, "RTP: A Transport Protocol for Real-Time Applications,” RFC 3550, (2003).

[7] H. Skinnemoen, R. Leirvik, J. Hetland, H. Fanebust and V. Paxal, “Interactive IP-Network Via Satellite DVB-RCS”, IEEE Journal on Selected Areas in Communications, vol. 22, pp.508-517, (2004).

[8] Z. Sun, "Satellite Networking: Principles and Protocols”, John Wiley \& Sons, (2005).

[9] Alex Vallejo, et. al., "State of the Art of IPv6 Conformance and Interoperability Testing,” IEEE Communications Magazine, pp.140146, (2007).

[10] BitTornado. [Online]. Available: http://www.bittornado.com

[11] Digital Video Broadcasting(DVB), Framing structure, channel coding and modulation for 11/12 GHz satellite services, ETSI EN 300 421, (1997).

[12] Digital Video Broadcasting(DVB); Interaction channel for Satellite Distribution Systems, ETSI EN 301 790, (2005).

[13] IST SATSIX Project. [Online]. Available: http://www.ist-satsix.org

[14] LFTP. [Online]. Available: http://lftp.yar.ru

[15] "Preparation for IPv6 in Satellite Communications: IABG," ESA Contract 17629/03/NL/ND, (2004).

[16] VLC media player. [Online]. Available: http://www.videolan.org/vlc

[17] Wireshark. [Online]. Available: http://www.wireshark.org. 